\title{
LAS EXPLOTACIONES FAMILIARES EN EL AGRO PAMPEANO: CONTROVERSIAS Y PERSPECTIVAS ${ }^{1}$
}

\author{
Clara Cravioti \\ craviotti@conicet.gov.ar \\ Investigadora independiente \\ Consejo Nacional de Investigaciones Científicas y Técnicas \\ Argentina
}

\section{RESUMEN}

Este artículo revisita las dimensiones constitutivas del debate acerca de la importancia y las características de la producción familiar en la región pampeana argentina a partir de trabajos académicos representativos de las diferentes corrientes analíticas, e identifica algunos nudos controversiales de las mismas. Posteriormente, aborda la problemática del carácter de las vinculaciones en las que participa este sujeto social y su grado de anclaje territorial, una temática que supone la reconsideración de aportes previos bajo una mirada más actual, que comprende tendencias recientes de la evolución de los sistemas agroalimentarios en el contexto de la globalización.

Palabras clave: producción familiar, redes sociales, desarrollo local, región pampeana.

\section{ABSTRACT}

This article revisits the constitutive dimensions of the debate about the importance and nature of family farming in the Pampas region of Argentina taking into account representative academic contributions coming from different analytical streams, identifying some of the controversial issues they raise. Then it considers the kind of linkages in which this social subject participates as well as its degree of territorial anchoring, a topic that involves the review of previous contributions by adopting a contemporary view that contemplates recent trends of evolution of the agrifood systems in the context of globalization.

Keywords: family farming, social networks, local development, Pampas region. 


\section{INTRODUCCIÓN}

La vigencia de la producción familiar ha sido una preocupación clásica de las ciencias sociales agrarias, que desde diversos abordajes teóricos han debatido acerca de su capacidad de persistir en un contexto de expansión de las modalidades capitalistas de producción. Las peculiaridades del agro que operan como frenos al desarrollo del capitalismo, y las especificidades de estas explotaciones en cuanto a racionalidad y retribución del trabajo han constituido la base de argumentaciones referenciales en la disciplina (Chayanov, 1974; Kautsky, 1980; Mann y Dickinson, 1978; Friedmann, 1981; Reinhardt y Barlett, 1987). Perspectivas más actuales ponen el acento, en cambio, en su habilidad para desarrollar alternativas productivas y de mercado que puedan transformarse en germen de cambio del paradigma productivo hegemónico —basado en la intensificación productiva y la ampliación de escala - así como en su participación en redes horizontales, con implicaciones positivas sobre la sustentabilidad de estas explotaciones y el desarrollo local (Van der Ploeg et al., 2004).

Tradicionalmente, el interés por esta forma productiva basada en la combinación de tierra y trabajo familiar obedece a su capacidad para contribuir a la seguridad alimentaria, la ocupación del territorio, la generación de empleo y la democratización de las estructuras económico-sociales, por lo que suele ser vista como un tipo deseable de organización social (Carballo, 2000). Expresiones políticas regionales y visiones ideológicas de la sociedad han tenido como punto de partida a la producción familiar y sus formas asociativas (Barsky, 1992). Más recientemente se constata una revitalización de la atención hacia este actor tanto desde el punto de vista académico como en materia de propuestas de intervención, que relegitima así la potencialidad de una forma productiva que el pensamiento clásico condenaba a desaparecer sin remedio.

En ese contexto, Argentina presenta especificidades con respecto a otros países latinoamericanos, en razón de su urbanización temprana y su base campesina más limitada. En particular en la región pampeana — conformada por la provincia de Buenos Aires, el 
oeste de La Pampa y el sur de las provincias de Entre Ríos, Córdoba y Santa Fe- la producción familiar se ha desarrollado desde sus inicios con una fuerte vinculación con los mercados de productos y de factores, adquiriendo la producción de autoconsumo escaso significado.

Dada la temprana integración del país en los mercados internacionales de granos basada en la extraordinaria fertilidad de las tierras pampeanas, la discusión sobre esta modalidad productiva ha estado inseparablemente ligada al debate sobre las características que asumía el modelo de desarrollo agropecuario pampeano en el transcurso de las diferentes etapas de su evolución. Con setenta millones de hectáreas, esta región es y ha sido el espacio más importante de la producción agropecuaria nacional, aporta actualmente el $80 \%$ de las exportaciones agropecuarias (Banco Mundial, 2006).

Teniendo en cuenta estos aspectos, el presente artículo revisita las dimensiones constitutivas del debate acerca de la importancia y las características de la producción familiar en el agro pampeano a partir de trabajos representativos de las diferentes corrientes analíticas, e identifica algunos nudos controversiales de las mismas. Después, aborda el carácter de las vinculaciones en las que participa este sujeto social y su grado de anclaje territorial, una temática que supone la reconsideración de aportes previos de otros autores bajo una mirada más actual sobre la evolución de los sistemas agroalimentarios en el contexto de la globalización. En este marco se asume la presencia de tendencias hacia la deslocalización de la producción agropecuaria, materializadas en rupturas entre el lugar de producción y los espacios de residencia, consumo e inversión (Hervieu, 1996; Bisang et al., 2008), así como también tendencias en contrario, que se expresan ya sea en acciones de resistencia individuales $\mathrm{y} / \mathrm{o}$ colectivas, como en comportamientos orientados al reforzamiento de las ventajas específicas locales. 


\section{LAS POSTURAS SOBRE LA VIGENCIA DEL PRODUCTOR FAMILIAR PAMPEANO}

La temprana incorporación de la Argentina como proveedora de granos para el mercado

mundial, basada en la figura central del «chacarero» ${ }^{2}$ como productor directo, dio lugar a diferentes intentos orientados a su caracterización (ya sea como «campesino», «campesino de tipo capitalista», «pequeño productor mercantil», «capitalista rural», entre otras), que han permeado la historiografía pampeana y continúan, en la actualidad, en un contexto redefinido a partir de los profundos cambios experimentados por el sector agroalimentario y sus relaciones con el conjunto de la sociedad.

Un denominador común de las diferentes caracterizaciones del «chacarero» de las primeras décadas del siglo XX es el énfasis puesto en las relaciones de producción al interior de la unidad productiva (en especial, en el peso del trabajo familiar frente al asalariado) y/o en el carácter — subordinado- de las vinculaciones que establecía con otros actores centrales de la producción agraria (terratenientes, empresas colonizadoras, comerciantes cerealistas y de ramos generales, etc.). Los enfoques que prestan atención al universo cultural y a las definiciones que de sí mismos hacían los «chacareros» han argumentado, sin embargo, que para hablar de la identidad de este sujeto social no solo hay que considerar su modo de acercamiento a la tierra y determinar las relaciones sociales que se gestaron en torno a la misma, sino también debe apelarse al universo cultural que opera como su espacio de pertenencia y de referencia, en el que se autodefine y se diferencia de otros actores (Bonaudo y Sonzogni, 1998). En esta línea, Ansaldi (1998) considera al «chacarero» como una categoría histórica propia del capitalismo agrario argentino, y como categoría analítica con identidad de clase «incompleta», obstruida por procesos de movilidad social ascendente. $^{3}$ Más recientemente, Balsa (2006) también pone el acento en aspectos culturales, al indicar que el concepto de «chacarero", si bien puede englobar posiciones de clase diferentes», resulta útil para dar cuenta de un modo o estilo de vida vigente durante el periodo de expansión agraria pampeano de comienzos del siglo XX. 
Este modo de vida se caracterizaba por la austeridad, el ahorro y el trabajo, para poder invertir los excedentes obtenidos en maquinarias o en tierras (arriendo o compra). Las mujeres realizaban tareas domésticas y estaban vinculadas al autoconsumo, pero raramente se dedicaban a labores ligadas a la producción de granos, las cuales estaban a cargo de los miembros varones de la familia; tenían actividades complementadas con el trabajo de terceros en las labores de cosecha.

Cabe señalar que aún para esta etapa —-donde las diferencias en materia de condiciones productivas y acceso a los recursos no eran demasiado notorias - algunas aproximaciones indicaban que resultaba problemático englobar las diferentes categorías de productores familiares bajo un concepto homogéneo (Pucciarelli, 1986; Bonaudo y Sonzogni, 1998). Estas especificidades iban más allá de la conocida distinción entre chacareros arrendatarios (predominantes en la estructura agraria pampeana de las primeras décadas del siglo) y colonos propietarios, emergentes de las diferentes iniciativas de colonización, tanto públicas como privadas.

El periodo que se inicia a principios de los años cuarenta del siglo pasado y culmina en los setenta da lugar a interpretaciones convergentes, centradas en la conversión del otrora «chacarero» en un farmer, similares al tipo norteamericano (Forni y Tort, 1991). Si bien se alude a la existencia de un proceso de expulsión de arrendatarios y recuperación de las propiedades arrendadas por parte de sus dueños (Slutzky, 1968; Llovet, 1986), y a la conversión en contratistas de servicios de maquinaria de parte de aquellos que no pudieron adquirir las tierras que trabajaban, cobra fuerza la interpretación de que el acceso a la propiedad de una buena proporción de los arrendatarios - aunado a un proceso de mecanización sustitutivo de mano de obra- posibilitó el citado cambio de categoría. Aunque no deja de mencionarse la paradoja que supone la emergencia durante esta etapa de una unidad familiar más «pura» en función de la casi ausencia de contratación de asalariados, pero más «capitalista» en términos del peso relativo de los factores de producción (Barsky, 1992). 
Los años noventa, signados por cambios marcados tanto en lo macroeconómico como en lo sectorial, al conjugar la política de apertura económica, desregulación y sobrevaluación de la moneda argentina bajo el «Plan de Convertibilidad» con el desarrollo de innovaciones técnicas (la más destacada fue el paquete tecnológico empleado en el cultivo de soja, basado en la combinación de variedades transgénicas, siembra directa y herbicida glifosato), inauguraron una nueva mirada sobre la explotación familiar pampeana. Una selección sobre el corpus de trabajos realizados permite establecer una distinción general entre aquellos que enfatizan los cambios producidos en la estructura interna de las explotaciones y quienes combinan estas transformaciones con las operadas en el plano cultural e ideológico.

Así, en la etapa inicial donde se perfilaban estas dinámicas —finales de los años noventa—, se pone el acento en los profundos cambios producidos en la dimensión «trabajo» de estas explotaciones, en tres planos que se afectaban mutuamente: a) la recurrencia a prestadores de servicios (contratistas de maquinaria), para llevar a cabo tareas del proceso productivo; b) el cambio cualitativo vinculado a la complejización del trabajo de gestión, y c) la pluriactividad o inserción en actividades fuera del predio. El primero de estos planos es considerado central, en tanto la expansión de la técnica de siembra directa llevó a que buena parte de los productores familiares optaran por contratar la labor de siembra que antes realizaban ellos mismos, ya que los equipos de que disponían se habían vuelto obsoletos y los nuevos tenían un costo elevado. Esta «externalización» de tareas profundizaba la tendencia preexistente a contratar ciertas labores (como la fumigación y la cosecha), al tiempo que permitía cuestionar la caracterización de los productores como familiares, en función de la presencia de trabajo doméstico en la ejecución de etapas centrales del proceso productivo (Craviotti, 2000). En este sentido, el trabajo mencionado propone que este nuevo tipo de productor familiar tenga a su cargo la gestión de un patrimonio familiar, supervise las operaciones cotidianas llevadas a cabo en la explotación y reserve para sí mismo la ejecución directa de las tareas críticas, procurando preservar el «lugar» de la familia en la reproducción de la explotación. 
En esta línea de preocupación por las transformaciones en las modalidades de trabajo, otros autores han inferido que la profundización de la contratación de servicios de maquinaria determina una transformación en la naturaleza social de los productores familiares, que indirectamente pasarían a apropiarse de la plusvalía generada por el trabajo ajeno (Martínez Dougnac, 2008). «Porque ¿qué queda de la personalidad “campesina” de las diferentes fracciones de "chacareros" en la medida en que reemplacen... el trabajo personal/familiar por la contratación de los diversos servicios agrícolas?» A pesar de que estos comportamientos han sido adoptados por productores familiares descapitalizados, éstos se han «aburguesado, aunque haya sido por necesidades que surgieron de estrategias de supervivencia campesina» (Azcuy y Fernández, 2008: 8).

Otra corriente conjuga estas transformaciones en la organización del trabajo con las mutaciones producidas en el plano cultural. Así, el trabajo de Balsa (2006) alude a las implicaciones de un fenómeno que empieza a profundizarse a partir de la década de los sesenta: el establecimiento de los familiares de los productores en las ciudades cabecera de los departamentos (ya que sólo una minoría había migrado a pequeñas localidades), que continúan haciendo el traslado cotidiano a las explotaciones. Este hecho conduce progresivamente a cambios en sus pautas de consumo (la mercantilización de los bienes consumidos y la adopción de pautas de diferenciación social, materializadas en un importante chalé urbano y la camioneta como signo de ostentación), así como a una menor socialización de los hijos en las tareas agrarias. Este proceso, calificado como de «aburguesamiento»y adopción de una cultura «sesgada» «por importantes elementos rentísticos», está, sin embargo, enraizado en el cambio producido por la posición social de estos productores, antes susceptibles de ser categorizados como «pequeño-burgueses con elementos campesinos», hoy como «pequeños capitalistas».

Por su parte, Gras (2009) indica que en la última década se ha producido el desplazamiento de una forma histórica de agricultura familiar en la región pampeana, que incluye tanto la expulsión de productores como un proceso de recomposición de los persistentes, en cuanto a su perfil socio-productivo. El autor identifica así los rasgos centrales de una novedosa 
capa de «empresarios familiares», que se caracterizan por combinar tierras propias y alquiladas a terceros (pequeños productores familiares que se convirtieron en rentistas), la externalización de una o más etapas del ciclo productivo y el mayor tiempo dedicado a las tareas de gestión. Asimismo, la profesionalización del oficio y un manejo de la explotación basado en la recurrencia al conocimiento «experto» se combinan con el predominio de una racionalidad de tipo formal. Estos empresarios familiares son definidos alternativamente como una fracción «que se distingue del resto de las capas familiares capitalizadas», o bien indicativa de la «formación de otro tipo de sujeto social»; una nueva burguesía agraria.

Matizando el tenor de estas argumentaciones, otros autores como Cloquell (et al., 2005) sostienen que aunque las transformaciones ocurridas a partir de los años noventa han provocado un fuerte impacto en materia de reducción de las explotaciones familiares y polarización de la estructura agraria pampeana, la organización laboral de las unidades que logran permanecer sigue siendo predominantemente familiar. En este sentido, un rasgo que destacan es que, durante los periodos clave de producción, todos los miembros participan indistintamente en los requerimientos del proceso.

Quizá la falta de cristalización de los procesos estudiados explique ciertas ambigüedades en las posturas asumidas por los autores. En realidad, en un conjunto importante de trabajos no existe acuerdo sobre el grado de «reversibilidad» de estas dinámicas si las condiciones coyunturales se modifican. Además, cabe agregar que gran parte de las argumentaciones se sustentan en estudios efectuados en zonas de agriculturización consolidada, por lo que el análisis del alcance de estos fenómenos en áreas marginales de la región, o bien en aquellas con presencia de actividades ganaderas, constituye una tarea aún pendiente. ${ }^{4}$

No obstante estas salvedades, parece tomar cuerpo la idea de que la producción familiar de la región pampeana se ha «empresarializado», desdibujándose su especificidad frente a los sectores definidamente capitalistas y perdiendo también su anclaje en la ruralidad, y en alguna medida en el territorio (esta cuestión será retomada en el apartado siguiente). Paralelo a estos procesos comienza a imponerse como autodefinición de estos actores el 
vocablo «productor» -desplazando al de «chacarero», que pasa a ser asociado a una forma de producción “perimida”. Esta forma de autodenominarse habría tomado fuerza a partir del proceso de modernización de la producción pampeana iniciado en los años sesenta, que remplazó bajo un tono pretendidamente neutral las denominaciones anteriores, borrando así las diferencias existentes entre ellos en cuanto a acceso a recursos, formas de organizar la producción, origen, etcétera (Albaladejo y Bustos Cara, 2008).

Por otra parte, en contraste con la etapa que va desde los años cincuenta a los ochenta —quizá la era de «oro» del productor familiar pampeano—, la etapa de finales del siglo XX comparte con la inaugurada en las primeras décadas del siglo XIX la idea de que la ahora transformada explotación familiar pampeana varía de acuerdo con el contexto general en que se inscribe. Si antiguamente la oposición estaba basada en la pequeña y mediana explotación (agrícola) versus la gran propiedad terrateniente (ganadera), en el periodo más inmediato parece ir cobrando forma un nuevo dualismo en círculos académicos y políticos -no sólo en la propia región pampeana sino también en la Argentina en su conjunto- entre la producción familiar como modo de vida y trabajo y el mundo de los denominados «agronegocios».

En el contexto argentino este último concepto alude a distintas dimensiones que empíricamente se presentarían combinadas con diferente grado e intensidad, tales como:

a) La creciente penetración de la producción agropecuaria por capitales provenientes de fuera del sector, que controlan su desarrollo (o incluso lo subordinan);

b) La orientación de la actividad agraria con base en oportunidades comerciales (principalmente vinculadas a la exportación) y hacia la búsqueda de beneficios rápidos, sin tomar en consideración otros criterios;

c) La recurrencia a prestadores de servicios para la realización de tareas del ciclo productivo, adoptando un modelo flexible de organización;

d) La conformación de redes para llevar a cabo la producción que, según el enfoque adoptado, pueden llegar a incluir cierta multiplicidad de agentes -aunque con diferente acceso a 
recursos y posición al interior de la red- o bien estar basadas exclusivamente en diferentes fracciones de capital;

e) La intensidad en el uso de insumos industriales y de tecnologías orientadas, ya sea a reemplazar mano de obra, atenuar la especificidad (biológica y climática) del agro, o a facilitar el seguimiento «a distancia» de las producciones desarrolladas;

f) La valorización del acceso a información técnica y de negocios, y de la capacidad de gestión por sobre los factores de producción «tradicionales» (como la tierra y el trabajo físico). Sin embargo, esto no significa que el grado de acceso al capital se transforme en un aspecto secundario, ya que este tipo de modelo productivo requiere de una fuerte disponibilidad de capital circulante;

g) La búsqueda de estandarización del paquete tecnológico empleado, que desvirtúa los saberes aprendidos y el «oficio» del productor frente a los componentes codificados de la nueva «profesión» de productor;

h) La descontextualización de la producción agraria, que se desarrolla sin tener en cuenta las especificidades de los espacios rurales tanto desde el punto de vista agroecológico como humano, la cual se independiza de la necesidad de velar por la sustentabilidad de éstos en el mediano y largo plazos.

El otro polo de este nuevo dualismo se conforma en el plano de la organización de los actores, principalmente aglutinados en el Foro Nacional de la Agricultura Familiar (FONAF). En el terreno discursivo se expresa en la construcción de la categoría «agricultura familiar», que es definida como una «forma de vida y una cuestión cultural», cuyo principal objetivo es «la reproducción social de la familia en condiciones dignas, donde la gestión de la unidad productiva y las inversiones en ella realizadas son hechas por individuos que mantienen entre sí lazos de familia, la mayor parte del trabajo es aportada por sus miembros, la propiedad de los medios de producción (aunque no siempre la tierra) les pertenece, y es en su interior que se realiza la transmisión de valores, prácticas y experiencias» (FONAF, 2006: 4). Apunta asimismo a incluir bajo la denominación de agricultor familiar a una multiplicidad de agentes -campesinos, chacareros, colonos, medieros, productores rurales sin tierra y comunidades de pueblos originarios. Sin entrar en una discusión que excedería los alcances de este trabajo, se puede sostener que 
ésta es una definición con fuertes componentes cualitativos, que enfatiza una marcada diferenciación entre el mundo rural y urbano, lo cual resulta difícil de sostener para ciertas áreas de la Argentina, particularmente la región pampeana. Esta definición conceptual es también complementada con la identificación de cinco categorías de agricultores familiares que son distinguidas con base en indicadores cuantitativos. No obstante, la correspondencia de tales indicadores con los componentes presentes en la definición conceptual no resulta del todo evidente o presenta problemas (Tsakoumagkos y Maraschio, 2009).

Si el vocablo «agricultura familiar» se constituye como construcción política que apunta a establecer una denominación más precisa de lo que se entiende ya no exclusivamente como una forma de organizar la producción, sino como un modo de vida. En la misma dirección operan otros esfuerzos encaminados a la ampliación «hacia abajo» del mismo concepto de productor, para dar cabida a sectores que no detentan la propiedad de la tierra pero sí trabajan en ella. Esta visión es promovida desde las escuelas rurales de alternancia, que nuclean principalmente a hijos de asalariados y pequeños productores agropecuarios en la provincia de Buenos Aires. ${ }^{5}$

Nosotros proponemos que son todos productores, sean empleados, sean medieros porque todos están trabajando en el suelo, en la tierra, también en la producción primaria, pero posicionándose en distintos lugares. Uno con el capital, otro con el trabajo (...) No "productor" por ser dueño del campo, se es productor aunque se sea mediero, el que me meto en la fosa, productor que me subo al tractor y sé que no es mío. (Director de escuela CEPT, 2007)

La polarización de agricultura familiar y agronegocios tiene, a nuestro juicio, la fortaleza y la debilidad de toda visión sintetizadora: fortaleza que radica en su capacidad de ilustrar ciertas tendencias destacadas de la evolución de formas de producción pampeanas, ahora extendidas a otras regiones de la propia Argentina; debilidad, al minimizar la 
heterogeneidad de situaciones existentes, que el desarrollo del capitalismo en el agro no ha logrado eliminar (Muráis, 1998; Tsakoumagkos et al., 2009).

\section{EL CHACARERO Y EL ESPACIO LOCAL}

Un elemento destacado de las distintas conceptualizaciones sobre la evolución de la producción familiar pampeana, es el supuesto implícito de que las recomposiciones experimentadas por ésta generan consecuencias significativas para la estructura social agraria y el sistema productivo en su conjunto. Sin embargo, los vínculos establecidos con otros actores -especialmente en el espacio local y en términos generales, la temática de la sociabilidad rural- han sido objeto de limitadas reflexiones por parte de los investigadores.

Es así como Taylor (1948) presta atención a elementos hoy comunes en los abordajes sobre desarrollo local, como es el caso del carácter de las vinculaciones entre los agentes y la importancia de la vecindad como matriz constitutiva de tales relaciones. Su trabajo empírico le permite sostener, para el momento de su estudio, que la sociabilidad entre los productores familiares pampeanos no era sistemática ni involucraba a todos los integrantes de las familias. ${ }^{6}$ En este sentido, resalta un conocimiento interpersonal reducido entre los vecinos producto de los cortos periodos de ocupación de las chacras, en el contexto de la vigencia del sistema de arrendamiento trianual. Además de la distancia física entre las explotaciones, existía una considerable distancia cultural y social entre productores y otras categorías sociales, que se evidenciaba en la casi ausencia de vínculos verticales. ${ }^{7}$ En su perspectiva, chacareros y residentes de los pueblos no eran parte de la misma comunidad en sentido social; las pequeñas localidades no constituían espacios de intercambio para los productores, ya que a ellas concurrían con escasa frecuencia. Organizaciones específicas como las cooperativas desempeñaban funciones básicamente económicas (eran business organizations) y sus gerentes no tenían un origen rural; mientras que el resto de las instituciones locales no requerían o permitían la participación de la comunidad. No estaban dadas entonces las condiciones para la emergencia de la acción colectiva local. 
Scobie (1968) retoma posteriormente esta línea de análisis al indicar que, en las primeras décadas del siglo XX, el aislamiento era la característica predominante de la escena rural argentina. Las chacras y puertos estaban unidos entre sí por los ferrocarriles -la forma empleada para transportar la producción de granos-, pero los eslabones de enlace no vinculaban a las chacras entre sí, ni a éstas con las comunidades o poblados rurales; las casas estaban dispersas y separadas unas de otras por distancias considerables. ${ }^{8}$ No obstante, esta interpretación es parcialmente cuestionada por Gallo (1983), quien adopta una mirada procesual al indicar que el aislamiento de los agricultores fue disminuyendo a medida que se expandían los cultivos cerealeros, no sólo gracias al desarrollo de los ferrocarriles y las localidades a él vinculadas o a la posesión de vehículos de transporte, sino también por la creación de escuelas e iglesias y el surgimiento de algunos periódicos locales.

La etapa que comienza a principios de los sesenta, signada por la modernización agropecuaria, la conformación del farmer y su radicación en centros urbanos, habría dado lugar desde algunas perspectivas a la desaparición de la sociabilidad rural (aunque, como vimos, ésta no parece haber sido demasiado rica en sus expresiones). ${ }^{9}$ En contrapartida, también habría abierto la puerta para otros intercambios de base urbana (como el bar, el club, el centro de acopio de cereales), que viabilizan el acceso a información y a oportunidades de negocios. Está implícito en esta visión que los otrora chacareros profundizan en este periodo sus vínculos verticales «hacia arriba», en desmedro de los vínculos «hacia abajo», por lo que estos últimos adquieren un carácter más impersonal y distante. Por otra parte, ya en la última década del siglo XX se perfilaría el dinámico sector de los «empresarios familiares», caracterizado por su disposición a integrar redes con agentes externos al sector agropecuario (que les permiten obtener el capital necesario para ampliar la superficie trabajada, o bien participar en negocios no agropecuarios). El contacto con círculos «expertos» de saberes técnicos es asimismo privilegiado como vía de acceso a información calificada (Gras, 2009). No obstante, desde una mirada centrada en los espacios locales queda pendiente el análisis del carácter simétrico o no de los vínculos establecidos y de las instancias, a través de las cuales se construyeron estas nuevas redes, 
así como profundizar en quiénes comenzaron la iniciativa para la asociación, lo que supone analizar el proceso social que posibilitó tales intercambios.

Coincidiendo con que el cambio hacia una residencia urbana implica el pasaje a otro tipo de sociabilidad y modo de vida, Sili (2000) nos propone, en cambio, una mirada territorial sobre el fenómeno, argumentando que ésta se corresponde con una nueva división social entre los productores. Así, diferencia a quienes permanecen en el campo (normalmente los de menores recursos), a los que se trasladan al pueblo y a los grandes productores (quienes siempre vivieron en las grandes ciudades). La realidad de los espacios rurales pampeanos sería entonces la multiplicación de «fragmentos socioterritoriales», que incluyen productores con similares relaciones sociales, a partir de las cuales construyen sus sistemas de representación y de pertenencia, así como los esquemas tecnológico-productivos que les permitirán subsistir. No obstante, el autor destaca que existen articulaciones entre ellos, representadas por quienes pertenecen a más de un fragmento socioterritorial y por el rol «bisagra» de los productores «del pueblo», quienes por su nivel de capitalización suelen prestar servicios de maquinaria tanto a los «del campo» como a los «de la ciudad». Se deduce que estos «mediadores» entre diferentes mundos construyen un tipo de sociabilidad particular frente a aquellos que permanecen en el campo. Por supuesto, esta sociabilidad sigue manteniendo una cierta especificidad que la diferencia respecto a la de los productores «de ciudad», ya que las relaciones que estos últimos establecen con los residentes en pueblos y áreas rurales dispersas son fundamentalmente de tipo funcional o laboral (contratación de empleados o de algún servicio). ${ }^{10}$ Si bien la línea interpretativa resulta algo mecanicista, tiene la ventaja de corresponderse con representaciones intuitivas sobre aspectos distintivos del mundo rural y sus habitantes que siguen vigentes a pesar de los crecientes vínculos entre lo rural y lo urbano, y que nociones como la de «nueva ruralidad» procuran captar.

Si según la perspectiva reseñada la vecindad y el espacio rural como lugar de construcción de relaciones sociales siguen teniendo una ubicación central en los productores familiares residentes en el campo, Albaladejo y Bustos Cara (2008) llevan el argumento más lejos: el 
desarrollo de las redes «deslocalizadas» propias del agro pampeano actual no tiene por qué volver ineficaces a las redes «micro», que mantienen su importancia para la creación y adaptación de cambios en la actividad agropecuaria. También identifican procesos de descomposición «hacia abajo» de la producción familiar pampeana, evidenciados en la existencia de una categoría de «chacareros minifundistas». Estos productores viven en el campo y sus redes de sociabilidad no tendrían ese componente de distancia social que algunos autores como Balsa adjudican al mundo chacarero actual, ya que incluyen a trabajadores (peones y encargados) de los establecimientos. Asimismo, la dimensión local permitiría un cierto sentimiento de pertenencia y formas de acción colectiva que se despliegan o reavivan ante situaciones de crisis. Las interrogantes que surgen frente a estos planteamientos se enfocan sobre la posibilidad de las modalidades de organización y participación por superar la esfera sectorial, que parece ser la marca distintiva de la sociabilidad del productor familiar emergente en el proceso de modernización agraria. También se cuestionan por el peso relativo de la categoría de pequeño productor familiar y el carácter distintivo de sus estrategias productivas.

Vemos aquí que la temática de las heterogeneidades de la producción familiar adquiere un lugar central, no simplemente desde el lado de las relaciones de producción al interior de las unidades, sino también en el plano de los vínculos establecidos con otros actores, en cierta medida, traspasados por el lugar de residencia (campo-pueblo-ciudad). Consideramos además que la heterogeneidad de los espacios rurales pampeanos debe también considerarse, por su influencia sobre el carácter de las vinculaciones entre los actores. Ya que no es lo mismo la red urbana del norte de la provincia de Buenos Aires que la del oeste; la primera es más densa y las distancias entre ciudades de tamaño intermedio y los pueblos son más cortas.

Por otra parte, el territorio como matriz básica de relaciones debe ser complejizado introduciendo la temática de la pluriactividad, considerando el ámbito espacial donde se desenvuelven las ocupaciones extraprediales de estos productores, así como su carácter y grado de conexión con el sector agropecuario. Al referirse a Brasil, la antropóloga Carneiro 
(1997) establece una diferencia interesante de los tipos de redes según el carácter del productor. Señala que en el productor «tradicional» las redes se centran en el círculo de vecindad, confundiéndose con las de amistad y de parentesco; en cambio, los agricultores «modernizados» y buena parte de los pluriactivos mantienen relaciones más diversificadas y más frecuentes con entidades territoriales más amplias: redes de comercialización y sistemas de información técnica y de financiamiento de amplitud regional en el primer caso, y personas de clase media urbana en el segundo caso.

Un tercer aspecto tiene que ver con el grado de fortaleza de los vínculos de quienes permanecen en el campo, en un contexto donde se ha producido una marcada disminución de las explotaciones agropecuarias, en particular de aquellas de menor tamaño. ${ }^{11}$ Es posible plantear que este proceso acarrea consecuencias sobre la fragilización y fragmentación de las redes sociales (Elverdín et al., 2008). La consiguiente disminución de la población residente en ámbitos rurales opera en el mismo sentido. Aun así, cabe reparar en que su número sigue siendo significativo (sólo en la provincia de Buenos Aires reúne a unas 500,000 personas), representando el 9.8\% de la población provincial, si se excluye al conurbano de la ciudad de Buenos Aires (MAA, 2005).

Teniendo presente estas consideraciones algunos interrogantes nos parecen centrales para orientar futuros trabajos: ¿en qué tipo de redes participa la heterogénea producción familiar pampeana, con las redefiniciones que ha experimentado en las últimas décadas? ¿En qué medida éstas se apoyan en el espacio local y se orientan al aprovechamiento/defensa de los recursos endógenos? ¿Cuánto pesa en su conformación el factor identitario? ¿Hasta qué punto aquellos sectores de la producción familiar que persisten sin haber recurrido a los esquemas productivos y organizativos propios del modelo productivo hegemónico, se basan en redes para desarrollar estrategias de resistencia alternativas? 


\section{CONCLUSIONES}

Como se desprende del análisis previo, más allá de la cuestión central de la estructura agraria conformada y sus implicaciones para el desarrollo del capitalismo agrario pampeano, las características del productor familiar emergente de las transformaciones acaecidas en las últimas décadas del siglo XX -tanto desde el punto de vista productivo como en el plano del estilo de vida- inciden en el tipo de redes en las que participa y en su grado de anclaje en el territorio. No solo se trata de las condiciones materiales que hacen posible ciertos intercambios y restringen otros. También son relevantes aspectos identitarios, vinculados a las definiciones que de sí mismos hacen los actores, las cuales también comportan definiciones de los otros. En este sentido, y aunque la noción de «productor» se haya convertido en hegemónica —quizá debido a su capacidad para neutralizar diferencias internas-, existen distintos significados asociados a ésta, así como nuevas construcciones discursivas (como la de agricultura familiar), que surgen como respuesta a la heterogeneidad de situaciones existentes y/o a la necesidad de fortalecer otro tipo de agricultura en un contexto de expansión del paradigma de los agronegocios.

Es posible que la visibilidad de ciertos procesos — mecanización sustitutiva de mano de obra, externalización de tareas a través de contratistas de servicios, creciente residencia urbana de los productores - apunte hacia un profundo cambio en la naturaleza de los productores familiares pampeanos y borre su especificidad frente a los productores definidamente empresariales, oscurecen situaciones de persistencia de sectores que no recurren a los esquemas productivos y organizativos propios de este modelo, y se basen en redes locales para desarrollar estrategias de resistencia alternativas. Dichas redes son importantes, ya que a través de ellas se movilizan recursos, se configuran identidades y se consolidan —o redefinen - relaciones de poder con vistas a la modificación del orden social instituido.

De allí la necesidad de análisis que, sin perder de vista las tendencias más generales, profundicen en las expresiones particulares de la categoría «productor familiar» en 
territorios y producciones específicas, así como en las bases materiales e intangibles en las cuales se sustenta.

Desde este punto de vista, una cuestión que se plantea — por cierto nada accesoria — remite a cómo se cruza la diversidad de agentes sociales con la cuestión territorial. Ello no envía a las condiciones que permiten que el espacio local se convierta en marco instituyente de acuerdos sociales orientados hacia la mejora del bienestar de la población que en ellos vive y trabaja; al alcance y relevancia de las redes que procuran la preservación del anclaje territorial de la producción agraria; al grado de participación de los productores familiares en tales redes.

Consideramos que la comprensión sobre cómo se insertan los actores en realidades productivas y territoriales heterogéneas, así como sobre los procesos sociales a través de los cuáles se alteran o no las relaciones de fuerzas de los campos en los cuales estos se desempeñan, constituye un aspecto de particular importancia en el actual contexto de evolución de los sistemas agroalimentarios. Desde este punto de vista, deben formar parte de la futura agenda de investigación de la cuestión rural-agraria. 


\section{BIBLIOGRAFÍA}

Abramovay, Ricardo, 2006, «Para una teoría de los estudios territoriales», en Desarrollo Rural. Organizaciones, instituciones y territorio, compilado por Mabel Manzanal, Guillermo Neiman y Mario Lattuada (coord.), Buenos Aires, CICCUS, pp. 51-70.

Albaladejo, Christophe y Roberto Bustos Cara, 2008, «Algarrobo o el fin del pueblo chacarero», en Transformaciones globales y Territorios. Desarrollo rural en la Argentina, experiencias y aprendizajes, compilado por Pablo Bilella y Esteban Tapella, Editorial La Colmena, Buenos Aires, pp. 63-93.

Ansaldi, Waldo, 1998, «¡jalá que llueva! Una vez más sobre la propuesta de conceptualizar a los chacareros pampeanos», ponencia presentada en las XVI Jornadas de Historia Económica, Universidad de Quilmes, Bernal.

Azcuy Ameghino, Eduardo y Diego Fernández, 2008, «Causas, mecanismos, problemas y debates en torno al proceso de concentración del capital agrario en la región pampeana: 1988-2007», ponencia presentada en las V Jornadas de investigación y debate Trabajo, propiedad y tecnología en la Argentina rural del siglo XX, Universidad Nacional de Quilmes.

Balsa, Javier, 2006, El desvanecimiento del mundo chacarero. Transformaciones sociales en la agricultura bonaerense, 1937-1988, Universidad Nacional de Quilmes, Bernal.

Banco Mundial, 2006, «Argentina. Agricultura y Desarrollo rural: Temas claves», Informe No. 32763-AR, Banco Mundial, Buenos Aires.

Barsky, Osvaldo, 1992, «Explotaciones familiares en el agro pampeano: procesos, interpretaciones y políticas», en Explotaciones familiares en el agro pampeano, volumen I, Centro Editor de América Latina, Buenos Aires, pp. 7-42. 
Bisang, Roberto, Guillermo Anlló y Mercedes Campo, 2008, «Una revolución (no tan) silenciosa. Claves para repensar el agro en Argentina», en Desarrollo Económico, vol. 48, núm. 190-191, Instituto de Desarrollo Económico y Social, Buenos Aires, pp. 165-207.

Bonaudo, Marta y Elida Sonzogni, 1998, «La construcción histórica de un actor: el chacarero de la pampa santafesina 1850-1912», ponencia presentada en las XVI Jornadas de Historia Económica, Universidad Nacional de Quilmes, Bernal.

Carballo, Carlos, 2000, «Programas Actuales para productores familiares e instrumentos de políticas diferenciadas», ponencia presentada en el Congreso de Profesionales de Cambio Rural.

Carneiro, María José, 1997, «Ruralidade: novas identidades em construção», ponencia presentada en el XXXV Congreso Brasileiro de Economía e Sociologia Rural, Natal.

Chayanov, Alexander, 1974, La organización de la unidad económica campesina, Editorial Nueva Visión, Buenos Aires.

Cloquell, Silvia, Roxana Albanesi, Mónica de Nicola, Cristina González, Graciela Preda y Patricia Propersi, 2005, «La agricultura a escala y los procesos de diferenciación social», Revista Interdisciplinaria de Estudios Agrarios, núm. 23, Centro Interdisciplinario de Estudios Agrarios, Buenos Aires, pp. 35-57.

Craviotti, Clara, 2000, «Los procesos de cambio en las explotaciones familiares pampeanas: Tendencias en el trabajo agrario y dinámicas familiares», Cuadernos de Desarrollo Rural, núm. 45, Pontificia Universidad Javeriana, Bogotá, pp. 69-89.

Elverdín, Julio, Andrea Maggio y José Muchnik, 2008, «Procesos de localización/deslocalización de las actividades productivas: expansión sojera y retracción 
ganadera en Argentina, estrategias de los productores», ponencia presentada en el IV Congreso de la Red SIAL, Mar del Plata.

Forni, Floreal y María Isabel Tort, 1991, «De chacareros a farmers contratistas», Serie Documentos de trabajo, núm. 25, Centro de Estudios e Investigaciones Laborales, Buenos Aires.

Foro Nacional de la Agricultura Familiar -FONAF-, 2006, Lineamientos generales de políticas públicas orientadas a la elaboración de un plan estratégico para la agricultura familiar, Buenos Aires, mimeo.

Friedmann, Harriet, 1981, «The Family Farm in Advanced Capitalism: Outline of a Theory of Simple Commodity Production in Agriculture», ponencia presentada en la Annual Meeting of the American Sociological Association, Madison.

Gallo, Ezequiel, 1983, La pampa gringa. La colonización agrícola en Santa Fe (18701895), Editorial Sudamericana, Buenos Aires.

González, María del Carmen, coordinadora, 2005, Productores familiares pampeanos: hacia la comprensión de similitudes y diferencias zonales, Editorial ASTRALIB, Buenos Aires.

Gorenstein, Silvia, coordinadora, 2006, Diagnóstico y propuestas para la elaboración de una estrategia de desarrollo rural de la provincia de Buenos Aires, SAGPyA PROINDERMinisterio de Asuntos Agrarios de la provincia de Buenos Aires (mimeo).

Gras, Carla, 2009, «La agricultura familiar en el agro pampeano. Desplazamientos y mutaciones», en Trabajo agrícola. Experiencias y resignificación de las identidades en el campo argentino, compilado por Talía Gutiérrez y Juan Manuel Cerdá, Editorial CICCUS, Buenos Aires, pp. 17-40. 
Gras, Carla y Valeria Hernández, 2009, La Argentina rural. De la agricultura familiar a los agronegocios, Editorial Biblos, Buenos Aires.

Hervieu, Bertrand, 1996, «Agricultura y territorio: nuevas orientaciones de la política agraria», Revista Española de Economía Agraria, Ministerio de Agricultura, Pesca y Alimentación, España, pp. 167-191.

Kautsky, Karl, 1980, La cuestión agraria, Siglo XXI, México.

Llovet, Ignacio, 1986, Tenencia de la tierra y estructura social en la provincia de Buenos Aires, (1960-1980), Centro de Investigaciones sobre el Estado y la Administración, Buenos Aires.

López, María Canela, 2010, «Desarrollo local, autoempleo y políticas educativas: un acercamiento a los CEPT y CEA de la provincia de Buenos Aires», en La otra agricultura. Trayectorias y estrategias de microemprendedores pampeanos, coordinado por Clara Craviotti, Editorial Biblos, Buenos Aires, pp. 107-128.

Mann, Susan y James Dickinson, 1978, «Obstacles to the development of a capitalist agriculture», Journal of Peasant Studies, Vol. 5, núm. 4, Routledge, pp. 466-471.

Márquez, Susana, 2007, Un año del Foro. Crónica, realizaciones y perspectivas del ejercicio de diálogo político, desarrollada por el Foro Nacional de la Agricultura familiar, (mimeo).

Martínez Dougnac, Gabriela, 2008, «Subsistencia y descomposición. Notas sobre el devenir de la agricultura familiar pampeana», en Pasado y presente del agro argentino, compilado por Javier Balsa, Graciela Mateo y María Silvia, Ospital Ediciones Lumiere, Buenos Aires, pp. 571-586. 
Ministerio de Asuntos Agrarios de la provincia de Buenos Aires -MAA-, 2005, Nuestra provincia, nuestro campo, Ministerio de Asuntos Agrarios, La Plata.

Murmis, Miguel, 1998, «Agro argentino: algunos problemas para su análisis»,en Las agriculturas del Mercosur: el papel de los actores sociales, compilado por Norma Giarracca y Silvia Cloquell, Editorial La Colmena, Buenos Aires, pp. 205-248.

Ploeg, Jan Douwe van der, Johan Bouma, Arie Rip, Frits Rijkenberg, Flaminia Ventura y Johannes Wiskerke, 2004, «On regimes, novelties, niches and co-production», en Seeds of transition. Essays on novelty production, niches and regimes in agriculture, compilado por Johannes Wiskerke y Jan Douwe van der Ploeg, Royal Van Gorcum Press, Assen, pp. 1-30.

Pucciarelli, Alfredo, 1986, El capitalismo agrario pampeano, Hyspamérica, Buenos Aires.

Reboratti, Carlos, 2010, «Un mar de soja: la nueva agricultura en Argentina y sus consecuencias», Revista de Geografía Norte Grande, núm. 45, Pontificia Universidad católica de Chile, pp. 63-76.

Reinhardt, Norah y Peggy Barlett, 1987, «Family Farm Competitiveness in United States Agriculture: A Conceptual Framework», ponencia presentada en la Annual meeting of the Rural Sociological Society, Madison.

Sartelli, Eduardo, 1998, «Entre la esencia y la apariencia, clase y estructura. ¿Qué es un chacarero?», ponencia presentada en las XVI Jornadas de Historia Económica, Universidad de Quilmes, Bernal.

Scobie, James, 1968, Revolución en las Pampas: Historia social del trigo argentino: 18601910, Solar/Hachette, Buenos Aires. 
Sili, Marcelo, 2000, Los espacios de la crisis rural. Geografía de una pampa olvidada. Editorial Universidad del Sur, Bahía Blanca.

Slutzky, Daniel, 1968, «Aspectos sociales del desarrollo rural en la pampa húmeda argentina», Desarrollo Económico, vol. 8, núm. 29, Instituto de Desarrollo Económico y Social, Buenos Aires, pp. 95-135.

Taylor, Carl, 1948, Rural life in Argentina, Louisiana State University Press, Baton Rouge.

Tsakoumagkos, Pedro y Fernanda González Maraschio, 2009, «Unidades familiares pampeanas: Algunas implicaciones de distintas definiciones existentes en la Argentina actual. Un ensayo en el caso de San Andrés de Giles (Buenos Aires) », ponencia presentada en el 9 Congreso Nacional de Estudios del Trabajo, Buenos Aires.

\section{NOTAS}

\footnotetext{
${ }^{1}$ Este artículo se realiza en el marco del proyecto PICT 2008 No. 1025, financiado por la Agencia Nacional de Promoción Científica y Tecnológica, Argentina.

${ }^{2}$ El vocablo «chacarero» proviene de «chacra», palabra que comenzó a designar a finales del siglo XIX a las unidades agrícolas de entre 50 y 200 hectáreas, contrastando con las estancias, explotaciones ganaderas que por lo general tenían más de 1000 hectáreas de superficie.

${ }^{3}$ En su perspectiva, la categoría chacarero remite a un productor rural (fundamentalmente agricultor), arrendatario y/o mediero que empleaba fuerza de trabajo familiar y recurría esporádica o estacionalmente a asalariados, apropiándose de una masa de plustrabajo, transferido como renta al propietario de la tierra y/o acumulado. Nótese que el uso de ambas preposiciones (y/o) tiene implicaciones bastante diferentes en cuanto a las características del chacarero como clase. Sartelli (1998: 10) coincide con Ansaldi en la necesidad de situar al chacarero en un momento histórico específico, aunque en su caso enfatiza la existencia de un proceso de construcción política que tendió a negar u oscurecer las relaciones sociales de producción fundamentales (con los asalariados), ubicándolo dentro de la burguesía.

${ }^{4}$ Algunas excepciones a mencionar son los trabajos de González et al. (2005) y Albaladejo y Bustos Cara (2008), quienes incluyen casos de productores ganaderos en su análisis y destacan la diversidad de comportamientos de los actores en los años noventa.

${ }^{5}$ La provincia de Buenos Aires es la única del país que posee escuelas públicas (CEPT) co-gestionadas por el Estado y las comunidades locales basadas en el sistema de alternancia, que implica que el proceso educativo se estructura en periodos alternados entre la escuela y la vivienda de los alumnos (López, 2009).

${ }^{6}$ «Farm families visit with each other, meet each other at trading centers, and their children attend neighborhood schools. In many areas none of these contacts except those at schools are systematic and school contacts generally include only children. Trade-center contacts are restricted almost altogether to men» (Taylor, op.cit.:268). El patrón de asentamiento de los productores familiares en las chacras se asemejaba al típico de las praderas estadounidenses, diferenciándose del patrón de asentamiento europeo en aldeas. El autor contrasta además la situación de dicho país, con numerosos pueblos de entre 1,000 y 5,000 habitantes, con la
} 
situación argentina para esa época, caracterizada por un buen número de ciudades de entre 10,000 y 40,000 habitantes, y pequeños pueblos con pocas funciones económicas y casi inexistentes funciones sociales.

${ }^{7}$ «Otro aspecto de la pronunciada distancia social entre gente de la finca y otros, es la casi completa ausencia del contactos sociales verticales. Pocos muchachos y muchachas granjeros adquieren una profesión, existiendo una gran distancia entre los tipos de conocimientos y pensamientos, los cuales prevalecen en diferentes niveles de vida» (Taylor, 1948: 272). (Traducción propia)

${ }^{8}$ «El almacén general fue la única institución social en la Argentina rural....En muchos sentidos ocupó el lugar de la iglesia, la escuela, el club y la plaza, todo lo cual brillaba por su ausencia en las pampas». (Scobie, 1968: 62)

9 «Las colonias se despoblaron...Los lazos sociales ligados al ocio colectivo en el campo fueron disminuyendo» (Balsa, op.cit.: 209) «Lentamente, la vida urbana se fue convirtiendo en una necesidad en la medida en que quedarse en el campo significaba quedar fuera de las relaciones sociales, no sólo de las nuevas, sino también de las previas, ya que los amigos/vecinos se habían mudado también a la ciudad» (Balsa, op.cit.:232).

${ }^{10}$ Aunque no queda del todo claro en la conceptualización del autor cuál es la expresión espacial de las tres configuraciones delineadas (por momentos en su enfoque pesa más cierta concepción del mundo y cierto modo de vida que el lugar de residencia en sentido estricto).

11 Tomando a la provincia de Buenos Aires, el Censo Agropecuario del 2002 registra 51,058 EAP (Explotaciones Agropecuarias) con una superficie agropecuaria total de 25,787,364 hectáreas. Respecto al Censo de 1988 se constata una disminución relativa de más de 20,000 EAP y de casi un millón y medio de hectáreas. La baja de explotaciones puede ser explicada en más del $80 \%$ por la desaparición de las unidades de menos de 200 hectáreas (Gorenstein, 2006).

Fecha de recepción: 6 de diciembre de 2010.

Fecha de aceptación: 7 de diciembre de 2011. 